\title{
Estudo comparativo da força de preensão isométrica máxima em diferentes modalidades esportivas
}

\author{
Comparative study of maximum isometric grip strength \\ in different sports
}

\author{
Noé Gomes Borges Junior ${ }^{1}$ \\ Susana Cristina Domenech \\ Affonso Celso Kulevicz da Silva ${ }^{1}$ \\ Jonathan Ache Dias ${ }^{1}$ \\ Yoshimasa Sagawa Junior ${ }^{1}$
}

1 Universidade do Estado de Santa Catarina, Centro de Ciências da Saúde e do Esporte, Laboratório de Instrumentação, Florianópolis, SC. Brasil.

Recebido em 14/04/08 Revisado em 19/06/08 Aprovado em 24/10/08
Resumo - O objetivo deste estudo foi comparar a força de preensão isométrica máxima (Fmax) entre diferentes esportes e entre mão dominante (FmaxD) e não dominante (FmaxND). Participaram do estudo 29 atletas (homens) das modalidades de aikidô (AI), jiu-jitsu (JJ), judô (JU) e remo (RE) e 21 não atletas (NA). O teste de preensão consistiu na manutenção da força isométrica máxima em um dinamômetro de preensão manual durante 10 s. O posicionamento dos sujeitos foi o sugerido pela Sociedade Americana de Terapia da Mão (ASHT). Para análise dos dados, utilizou-se o teste ANOVA fatorial 2 X5 com correção de Bonferroni, seguido dos testes t pareado e de Tukey. Foi verificado que existem maiores valores de Fmax quando o esporte praticado é JJ e quando usam a mão dominante, seguido dos grupos de JU, RE, AI e NA. Foi detectado que 30,9\% da variação da Fmax pode ser atribuída à dominância das mãos, 39,9\% às diferenças entre as modalidades esportivas e 21,3\% à interação entre a dominância de mãos e grupo de indivíduos. Os resultados do presente estudo permitiram verificar diferenças significativas na Fmax somente entre os grupos de JJ e AI, e JJ e NA, tanto na mão dominante quanto na não-dominante, e entre mão dominante e não dominante somente nos grupos de AI e NA. Os resultados deste estudo indicam que a Fmax pode ser empregada para comparação entre diferentes modalidades esportivas, e entre mão dominante e não dominante. Estudos com maior número de indivíduos permitirão identificar diferenças entre outras modalidades.

Palavras-chave: Biomecânica; Força da mão; Esportes.

Abstract - The objective of this study was to compare maximum isometric grip strength (Fmax) between different sports and between the dominant $(F \max D)$ and non-dominant (FmaxND) hand. Twenty-nine male aikido (AI), jiujitsu (JJ), judo (JU) and rowing (RO) athletes and 21 non-athletes (NA) participated in the study. The hand strength test consisted of maintaining maximum isometric grip strength for 10 seconds using a hand dynamometer. The position of the subjects was that suggested by the American Society of Hand Therapy. Factorial 2 X5 ANOVA with Bonferroni correction, followed by a paired t test and Tukey test, was used for statistical analysis. The highest Fmax values were observed for the JJ group when using the dominant hand, followed by the JU, RO, AI and NA groups. Variation in Fmax could be attributed to hand dominance (30.9\%), sports modality (39.9\%) and the interaction between hand dominance and sport (21.3\%). The present results demonstrated significant differences in Fmax between the JJ and AI groups and between the JJ and NA groups for both the dominant and non-dominant hand. Significant differences in Fmax between the dominant and non-dominant hand were only observed in the AI and NA groups. The results indicate that Fmax can be used for comparison between different sports modalities, and to identify differences between the dominant and nondominant hand. Studies involving a larger number of subjects will permit the identification of differences between other modalities.

Key words: Biomechanics; Hand strength; Sports. 


\section{INTRODUÇÃO}

A força de preensão manual é a capacidade da mão em realizar tarefas, imprimir forças e segurar objetos por meio de um conjunto de vetores de forças e momentos aplicados a um ponto ${ }^{1-3}$. De maneira abrangente, os tipos de preensão manual são divididos em: "Power Grip", na qual existe a inibição da ação do polegar; "Key Grip", quando a força é exercida pelo polegar sobre o lado radial da falange média do indicador e as do tipo "Pinch Grip", ou preensão de pinça, sendo a força que o polegar exerce sobre a falange distal de cada um dos dedos ${ }^{4}$. O tipo de contração muscular na preensão é também um fator relevante na realização do movimento, podendo ser: isométrico contínuo - a contração ocorre continuamente por determinado tempo - ou intermitente - existe um intervalo de tempo entre contrações sucessivas ${ }^{4,5}$.

Em diversos esportes, as mãos são utilizadas em determinadas situações, com movimentos de alto grau de habilidade, força e resistência muscular, que unidos, permitem a obtenção de um bom desempenho ${ }^{6-8}$. Nestas modalidades, a força de preensão é uma importante característica do movimento das mãos. Tendo em vista que muitos esportes utilizam o movimento de preensão na prática esportiva, informações sobre esta característica são úteis para desenvolver protocolos específicos para o fortalecimento das mãos do atleta e a prevenção de lesões ${ }^{7,9}$. Estudos sobre a biomecânica da preensão manual, geralmente, são voltados a populações de nãoatletas, ou então para aquelas que possuem algum tipo de disfunção muscular, articular ou nervosa ${ }^{10-13}$. Pesquisas em diversas áreas têm gerado informações que podem, através de pequenas modificações no treinamento, promover um aumento no rendimento, levando o atleta à vitória ${ }^{14-16}$. Porém, até o momento, o conhecimento do comportamento da força de preensão manual em atletas não é muito aprofundado $^{17}$.

Verifica-se a necessidade da realização de estudos relacionados à avaliação dos diferentes parâmetros biomecânicos envolvidos na preensão manual (dentre estes a força máxima), em diferentes modalidades esportivas, de forma a gerar informações que permitam estabelecer avaliações quantitativas sobre o desempenho de atletas. Desta forma, poder-se-á auxiliar na prescrição de treinamentos, de acordo com a especificidade dos movimentos de cada esporte.

No intuito de gerar informações que permitam uma análise mais minuciosa sobre a biomecânica da preensão manual, em esportes que utilizam a mesma na prática esportiva, neste trabalho, avaliouse a força de preensão isométrica máxima (Fmax) em ambas as mãos de atletas de modalidades que utilizam a preensão manual na prática esportiva - aikidô, jiu-jitsu, judô, remo e um grupo de não-atletas. Objetivou-se verificar se é possível identificar diferenças entre as diferentes modalidades esportivas e entre mão dominante e não dominante, utilizando este parâmetro. Pretende-se obter informações nos diferentes grupos estudados, no intuito de, futuramente, propor protocolos de avaliação e treinamento específicos às modalidades estudadas.

\section{PROCEDIMENTOS METODOLÓGICOS}

Participaram do estudo, atletas profissionais com $25 \pm 5$ anos, homens, de modalidades que utilizam a preensão manual com predominância na sua prática, divididos em: Aikidô (AI, n=7), Jiu-jitsu (JJ, n=7), Judô (JU, n=8) e Remo (RE, n=7). Para comparação, foram também avaliados indivíduos não-atletas (NA, $n=21$ ), praticantes de algum tipo de exercício físico sem objetivo competitivo. Condições de inclusão: os sujeitos não deveriam apresentar problemas nas articulações das mãos, cotovelos e ombros, e os atletas deveriam ter um tempo de prática do esporte superior a um ano sem interrupção.

Para mensuração da força de preensão manual, foi utilizado um dinamômetro (Figura 1) desenvolvido no Laboratório de Instrumentação da UDESC. Este possui excelente coeficiente de linearidade $\left(\mathrm{r}^{2}=0,9999\right)$ e permite ajuste contínuo da empunhadura $(0,04-0,12) \mathrm{m}$. Para aquisição do sinal, foram utilizados um amplificador (ENDEVCO, modelo 136, EUA), e uma placa de aquisição de dados PC-CARD-DAS16/16-AO (Computer Boards, EUA) ligada a um computador. Para aquisição e armazenamento dos dados utilizou-se o software $\mathrm{SAD} 32^{\circledR}$, ajustando a freqüência de aquisição em $100 \mathrm{~Hz}$.

O protocolo de coleta de dados foi baseado em alguns estudos da literatura ${ }^{17-23}$. Para o posicionamento dos sujeitos (Figura 1), adotou-se o sugerido pela Sociedade Americana de Terapia da Mão (ASHT): os sujeitos permaneceram sentados com a coluna ereta, mantendo o ângulo de flexão do joelho em $90^{\circ}$. O ombro foi posicionado em adução e rotação neutra, o cotovelo foi flexionado a $90^{\circ}$, com antebraço em meia pronação e punho neutro, podendo movimentá-lo até $30^{\circ}$ graus de extensão. O braço manteve-se suspenso no ar com a mão po- 
sicionada no dinamômetro, este último sustentado pelo avaliador. A empunhadura utilizada foi de 0,055 m. Depois de posicionados, os sujeitos foram instruídos a realizarem a preensão manual com o máximo de força possível durante $10 \mathrm{~s}$, com flexão total do $2^{\circ}$ ao $5^{\circ}$ dedos sobre a região palmar, e inibição da ação do polegar (Power grip). Durante o tempo de coleta, o indivíduo foi incentivado verbalmente e não houve feedback visual sobre o seu desempenho. Os dados foram coletados, inicialmente, na mão dominante, e depois na nãodominante. $O$ protocolo foi aprovado previamente pelo comitê de ética local para pesquisa em seres humanos (processo 41/05).

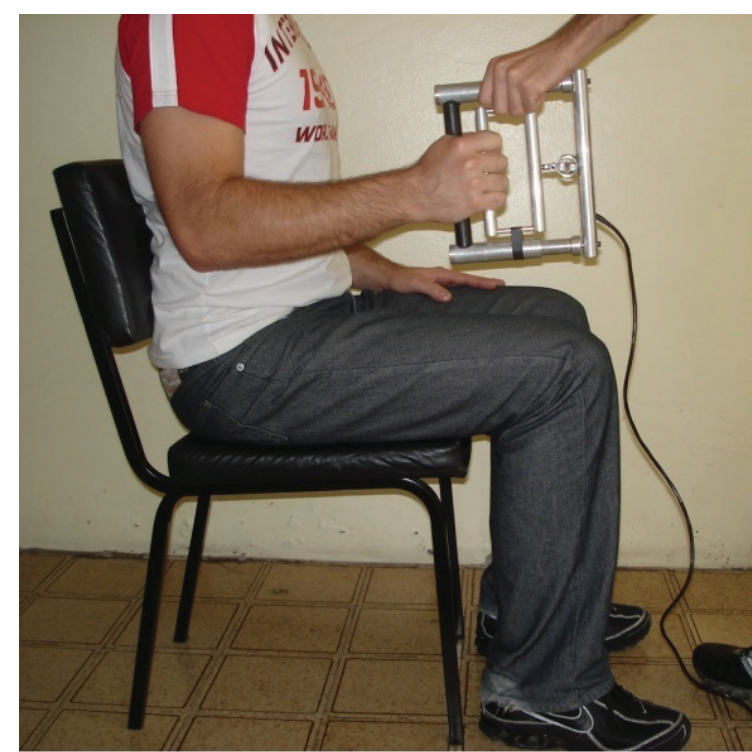

Figura 1. Posicionamento do sujeito durante a avaliação de preensão manual.

A análise estatística consistiu do cálculo de medidas descritivas, verificação dos pressupostos paramétricos por meio dos testes de Shapiro-Wilk, Levene, Razão Máxima e Mauchly. Para verificar o efeito do tipo de esporte praticado e da dominância das mãos sobre a força de preensão máxima, utilizou-se o teste ANOVA fatorial 2X5 (Splitplot ANOVA) com correção de Bonferroni. Para tanto, considerou-se uma variável independente entre grupos a cinco níveis (grupo de indivíduos, separados por tipo de esporte praticado) e uma variável independente intra-grupos a dois níveis (dominância das mãos), em relação à variável dependente (Fmax).

Para verificar as diferenças entre as condições de dominância em cada um dos grupos de indivíduos (efeitos simples), empregou-se o teste t pareado, e para verificar as diferenças entre os grupos de indivíduos, em uma das condições de dominância da mão (efeitos simples), empregou-se o teste de Tukey. Foi utilizado o software SPSS ${ }^{\otimes} 14.0$, adotando um nível de significância de 5\%.

\section{RESULTADOS}

Os intervalos de 95\% de confiança para a Fmax são ilustrados na Figura 2.

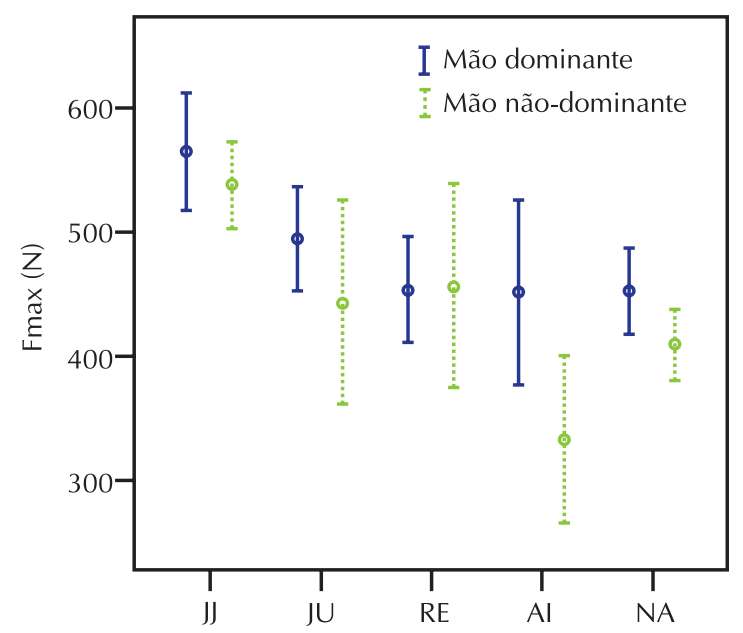

$J U=$ Judô; J = Jiu-jitsu; RE = Remo; $A I=$ Aikidô; $N A=$ nãoatletas.

Figura 2. Limites de confiança de 95\% em torno das médias de Fmax obtidas na mão dominante e não-dominante em diferentes modalidades esportivas.

A Anova Fatorial 2X5 foi executada e as hipóteses de normalidade, homogeneidade das variâncias e esfericidade foram satisfeitas. A Tabela 1 apresenta os valores dos efeitos principais do grupo de indivíduos, da dominância das mãos, da interação entre grupo de indivíduos e dominância das mãos sobre a Fmax.

Tabela 1. Valores da Soma dos Quadrados $(\Sigma Q)$, graus de liberdade $(\mathrm{gl})$, estatística F, probabilidade de significância (p) e tamanho do efeito $\left(\eta^{2}\right)$ para as diferentes fontes de variação.

\begin{tabular}{lccccc}
\hline Fonte de variação & $\Sigma Q$ & gl & $F$ & $p$ & $\eta^{2}$ \\
\hline $\begin{array}{l}\text { Grupo } \\
\text { de indivíduos }\end{array}$ & 211641,65 & 4 & 7,48 & $0,000^{*}$ & 0,399 \\
$\begin{array}{l}\text { Dominância } \\
\text { das Mãos }\end{array}$ & 47341,85 & 1 & 20,12 & $0,000^{*}$ & 0,309 \\
& & & & &
\end{tabular}

Interação Grupo

de indivíduos

vs Dominância

$28743,75 \quad 4 \quad 3,05 \quad 0,026^{*} \quad 0,213$

das mãos

$\begin{array}{ll}\text { Erro } & 105910,12 \quad 45\end{array}$

* significativo para $\mathrm{p}<0,05$. 
Tabela 2. Valores das diferenças entre as médias de Fmax $(\Delta \bar{x})$, erro padrão da média amostral (EPM), probabilidade de significância (p) e intervalo para 95\% de confiança (IC) calculados pelo teste de Tukey.

\begin{tabular}{|c|c|c|c|c|c|c|c|}
\hline \multirow[t]{2}{*}{ Dominância } & \multicolumn{2}{|c|}{$\begin{array}{c}\text { Grupos } \\
\text { de Comparação }\end{array}$} & \multirow[t]{2}{*}{$\Delta \bar{x}(\mathrm{~N})$} & \multirow[t]{2}{*}{$\operatorname{EPM}(\mathrm{N})$} & \multirow[t]{2}{*}{$\mathrm{p}$} & \multicolumn{2}{|c|}{ IC } \\
\hline & & & & & & Lim. Inf. & Lim. Sup. \\
\hline \multirow{10}{*}{ Dominante } & \multirow{4}{*}{ J } & J & 70,513 & 34,091 & 0,252 & $-26,353$ & 167,380 \\
\hline & & RE & 111,698 & 35,209 & $0,022^{*}$ & 11,654 & 211,741 \\
\hline & & $\mathrm{Al}$ & 113,183 & 35,209 & $0,019 *$ & 13,139 & 213,226 \\
\hline & & NA & 112,249 & 28,748 & $0,003^{*}$ & 30,564 & 193,934 \\
\hline & \multirow{3}{*}{ JU } & RE & 41,184 & 34,901 & 0,747 & $-55,682$ & 138,051 \\
\hline & & $\mathrm{Al}$ & 42,669 & 34,091 & 0,721 & $-54,197$ & 139,536 \\
\hline & & NA & 41,735 & 27,367 & 0,552 & $-36,026$ & 119,497 \\
\hline & \multirow{2}{*}{ RE } & $\mathrm{Al}$ & 1,485 & 35,209 & 1,000 & $-98,558$ & 101,529 \\
\hline & & NA & 0,551 & 28,748 & 1,000 & $-81,134$ & 82,236 \\
\hline & $\mathrm{Al}$ & NA & $-0,934$ & 28,748 & 1,000 & $-82,619$ & 80,751 \\
\hline \multirow{10}{*}{ Não-dominante } & \multirow{4}{*}{ J } & JU & 94,963 & 36,920 & 0,093 & $-9,944$ & 199,871 \\
\hline & & RE & 80,807 & 38,131 & 0,230 & $-27,541$ & 189,155 \\
\hline & & $\mathrm{Al}$ & 205,071 & 38,131 & $0,000^{*}$ & 96,723 & 313,419 \\
\hline & & NA & 128,864 & 31,134 & $0,001^{*}$ & 40,398 & 217,330 \\
\hline & \multirow{3}{*}{ JU } & RE & $-14,157$ & 36,920 & 0,995 & $-119,064$ & 90,751 \\
\hline & & $\mathrm{Al}$ & 110,108 & 36,920 & $0,035^{*}$ & 5,200 & 215,015 \\
\hline & & NA & 33,901 & 29,639 & 0,782 & $-50,316$ & 118,118 \\
\hline & \multirow{2}{*}{ RE } & $\mathrm{Al}$ & 124,264 & 38,131 & $0,017^{*}$ & 15,916 & 232,612 \\
\hline & & NA & 48,057 & 31,134 & 0,540 & $-40,409$ & 136,523 \\
\hline & $\mathrm{Al}$ & NA & $-76,207$ & 31,134 & 0,121 & $-164,673$ & 12,259 \\
\hline
\end{tabular}

* significativo para $\mathrm{p}<0,05$. JU = Judô; J = Jiu-jitsu; RE = Remo; AI = Aikidô; NA = não-atletas.

Tabela 3. Valores das diferenças entre as médias de $\operatorname{Fmax}(\Delta \bar{x})$ entre mão dominante e mão não-dominante nos diferentes grupos, desvio padrão (DP), erro padrão da média amostral (EPM), estatística t, graus de liberdade (gl), probabilidade de significância (p) e intervalo para $95 \%$ de confiança (IC) calculados no teste t pareado.

\begin{tabular}{|c|c|c|c|c|c|c|c|c|}
\hline Grupo & $\Delta \bar{x}(\mathrm{~N})$ & $\mathrm{DP}(\mathrm{N})$ & $\operatorname{EPM}(\mathrm{N})$ & $t$ & $\mathrm{gl}$ & $p$ & IC & \\
\hline & & & & & & & Lim. Inf. & Lim. Sup. \\
\hline J & 27,305 & 54,569 & 20,62497 & 1,324 & 6 & 0,117 & $-49,161$ & 103,770 \\
\hline RE & $-3,586$ & 84,723 & 32,02226 & 0,112 & 6 & 0,457 & $-122,307$ & 115,134 \\
\hline $\mathrm{Al}$ & 119,193 & 98,529 & 37,24046 & 3,201 & 6 & $0,009 *$ & $-18,873$ & 257,259 \\
\hline
\end{tabular}

* significativo para $\mathrm{p}<0,05$. JU = Judô; J = Jiu-jitsu; RE = Remo; AI = Aikidô; NA = não-atletas.

Tendo a interação apresentado significância estatística, foram verificados os efeitos simples. $\mathrm{Na} \mathrm{Ta}$ bela 2 , são mostrados os resultados do teste de Tukey e na Tabela 3, os resultados do teste t pareado.

Em relação às medidas descritivas (Figura 2), pode-se observar que os maiores valores de força foram encontrados no grupo de JJ $($ Fmax $\mathrm{D}=$
$564,9 \pm 18,9 \mathrm{~N} ;$ FmaxND $=537,6 \pm 14,1 \mathrm{~N})$, seguidos do grupo de JU (FmaxD = 494,4 $\pm 48,9 \mathrm{~N}$; FmaxND $=442,6 \pm 95,1 \mathrm{~N})$. Menores valores foram encontrados nos atletas de RE (FmaxD $=453,2 \pm 46,2 \mathrm{~N}$; FmaxND $=456,8 \pm 88,6 \mathrm{~N}), \mathrm{AI}(\mathrm{FmaxD}=451,7 \pm 79,1$ $\mathrm{N} ; \mathrm{FmaxND}=332,5 \pm 72,2 \mathrm{~N})$ e $\mathrm{NA}(\mathrm{FmaxD}=$ $452,6 \pm 75,2 \mathrm{~N} ; \mathrm{FmaxND}=408,7 \pm 62,8 \mathrm{~N})$. 


\section{DISCUSSÃO}

Por se tratar de uma pesquisa de cunho exploratório, uma análise comparativa dos resultados obtidos com os da literatura é de difícil execução, tendo em vista algumas limitações. Embora numerosos estudos sobre força de preensão manual tenham sido publicados, muitos resumem dados obtidos com instrumentos e procedimentos diferentes dos recomendados pela $\mathrm{ASHT}^{24}$. O uso de diferentes instrumentos, os quais são construídos dentro de concepções diferentes em termos de design, princípio de funcionamento e tipos de sensores, dificulta comparações diretas. Adicionalmente, um pequeno número de publicações aborda a preensão manual em populações de atletas ${ }^{7-9,25-29}$, sendo em sua maioria, focados em populações de não atletas ${ }^{1,5,10-12,17,19-24}$.

Os valores de referência citados em literatura ${ }^{24}$ de Fmax para homens de 20 a 25 anos são de 443,4 $\mathrm{N}$ a 603,3 N (FmaxD) e 380,6 N a 550,3 N (FmaxND) e para indivíduos de 25 a 29 anos, de 434,6 $\mathrm{N}$ a $623,9 \mathrm{~N}$ (FmaxND) e de 403,2 $\mathrm{N}$ a $577,8 \mathrm{~N}$ (FmaxND). Observa-se que todos os grupos avaliados no presente estudo apresentaram valores de força dentro desta faixa de valores.

Na mão dominante, em relação aos atletas de JJ (Figura 2), observou-se uma diminuição nos valores médios da Fmax de, aproximadamente, 12,5\%; $19,8 \% ; 20,0 \%$ e $19,9 \%$ para o grupo de JU, RE, AI e NA, respectivamente, e na mão não-dominante observou-se uma diminuição mais acentuada de, aproximadamente, $17,7 \% ; 15,1 \% ; 38,2 \%$ e $24,0 \%$.

Dentre os grupos de atletas, o grupo de JJ apresentou intervalo de confiança com menor amplitude (Figura 2), o que sugere ser o grupo mais homogêneo em termos de valores de força de preensão. O grupo de NA, apesar de apresentar valores menores de Fmax, apresentou-se homogêneo, com intervalo de confiança de amplitude próxima ao dos atletas de JJ. Porém, houve um aumento na amplitude do intervalo de confiança na mão não-dominante em relação à dominante, no caso dos atletas de JU, RE e AI. Estes resultados demonstram que, em geral, a mão dominante tem melhor desempenho, não apenas no teste de força máxima, mas também tem menor variabilidade dos dados. O grupo do RE, por sua vez, não apresentou praticamente nenhuma diferença entre FmaxD e FmaxND, apenas a mão dominante apresentou menor variabilidade. Este fato pode ser atribuído à utilização das duas mãos com a mesma intensidade e volume na prática deste esporte.
Comparando os resultados de Fmax obtidos com estudos da literatura, em estudo ${ }^{30}$ realizado no Brasil com atletas de JJ (24,5 $\pm 5,8$ anos), com tempo de prática no esporte de 3,5 $\pm 2,1$ anos, foram encontrados valores de 463,8 \pm 65,7 $\mathrm{N}$ para FmaxD e 444,2 \pm 45,11 N para FmaxND, os quais são menores do que aqueles encontrados no presente estudo para os praticantes de JJ. Claessens et al..$^{25}$, ao avaliar atletas belgas de JU de alto nível, encontraram valores de 636,4 \pm 87,3 $\mathrm{N}$ para FmaxD e 585,4 \pm $86,3 \mathrm{~N}$ para FmaxND, os quais se encontram acima dos valores médios medidos em todos os grupos do presente estudo. Little ${ }^{26}$ avaliou atletas Canadenses de judô, encontrando o valor médio de $565,8 \mathrm{~N}$ para Fmax, valor superior ao encontrado por Thomas et al. $^{27}$, que avaliaram atletas da equipe nacional do Canadá, encontrando o valor médio de 552,1 N para Fmax. Ambos os estudos demonstram valores acima dos valores encontrados para atletas de judô do presente estudo. Tsuji et al. ${ }^{8}$ avaliaram atletas amadores de Wrestlers, encontrando o valor médio de $505 \mathrm{~N}$ para FmaxD, próximo ao valores do grupo de JU do presente estudo. Outro estudo ${ }^{28}$ avaliando atletas de elite de uma arte marcial chamada Pencak Silat, encontrou valores de 435 \pm 79 N FmaxD, próximo aos encontrados nos grupos RE, AI e NA do presente estudo. Adicionalmente, em outro estudo $^{29}$, foram encontrados valores de 622 $\pm 20,9$ $\mathrm{N}$ para FmaxD e $591,4 \pm 20,7 \mathrm{~N}$ para FmaxND em atletas de cabo de guerra e $607,1 \pm 13,2 \mathrm{~N}$ para FmaxD e 568,2 $\pm 12,5 \mathrm{~N}$ para FmaxND em atletas de Rugby. Estes valores estão acima dos valores encontrados no presente trabalho, possivelmente, devido à diferença de treinamento e característica dos esportes. Vale salientar que comparações com a literatura mais detalhadas são dificultadas pelas limitações supracitadas.

A análise de variância fatorial revelou que os efeitos principais devidos aos fatores grupo de indivíduos, dominância das mãos e interação são improváveis de terem ocorrido somente devido ao erro amostral (Tabela 1). O efeito principal da variável grupo de indivíduos corrobora as afirmações anteriormente citadas, indicando que existem maiores valores de Fmax quando o esporte praticado é JJ e quando usam a mão dominante, seguido do grupo de JU, RE, AI e NA. Assim, 39,9\% da variação de Fmax se devem à manipulação da variável independente grupo de indivíduos. Além disso, 30,9\% da variação da Fmax foram atribuídos ao efeito da dominância das mãos. Finalmente, a interação entre a dominância de mãos e grupo de indivíduos foi considerável e representa 21,3\% da variância total. 
Segundo a Tabela 2, na condição mão dominante, foram identificados efeitos significativos entre os grupos JJ e RE ( $\mathrm{p}=0,022)$; JJ e AI ( $\mathrm{p}=0,019)$; JJ e NA ( $p=0,003)$. Já na condição mão não-dominante, foram encontrados efeitos significativos entre os grupos JJ e AI $(p<0,001)$; JJ e NA ( $p=0,001)$; JU e AI $(p=0,035)$; RE e AI $(p=0,017)$. Na Tabela 3, observaram-se diferenças significativas da Fmax entre mão dominante e não-dominante somente nos grupos de AI $(p=0,009)$ e NA $(p<0,001)$. A diferença dos valores de Fmax observada entre mão dominante e não-dominante nos atletas de $\mathrm{AI}$ pode ser explicada pelas características deste esporte, no qual existe predomínio das atividades com membro dominante, possuindo, geralmente, melhor coordenação e maior força. Por outro lado, no caso dos não atletas, a diferença de força observada entre membro dominante e não-dominante está de acordo com a literatura para este tipo de população $0^{24}$.

\section{CONCLUSÃO}

Os resultados do presente estudo permitiram verificar diferenças significativas nos valores de Fmax somente entre os grupos de JJ e AI, e JJ e NA, tanto na mão dominante quanto na mão não-dominante. Nas outras comparações, foi verificada diferença entre grupos somente em uma das mãos, não podendo, desta forma, identificar um efeito genuíno do tipo de esporte praticado sobre a Fmax.

Apesar do consenso entre os pesquisadores do tema que a mão dominante apresenta melhor desempenho que a mão não-dominante, isto foi verificado somente nos grupos de NA e AI. Nos demais atletas, este comportamento depende das características do esporte.

Desta forma, o presente estudo apresenta a possibilidade de utilizar o parâmetro Fmax para comparação entre diferentes modalidades esportivas, e entre mão dominante e não dominante, dentro do mesmo grupo. Acredita-se que as diferenças verificadas nos dados obtidos, serão possíveis de serem observadas em outras modalidades, à medida que estudos com maior número de atletas em cada grupo sejam realizados.

Portanto, é necessário que sejam desenvolvidas novas pesquisas, apontando valores de referência de Fmax, como o trabalho de Bohannona et $\mathrm{al}^{24}$, voltados à população de atletas, levando em consideração aspectos anatômicos, mecânicos e do treinamento, a fim de melhor caracterizar esta importante capacidade das mãos e criar parâmetros para avaliação de desempenho.

\section{REFERÊNCIAS BIBLIOGRÁFICAS}

1. An KN, Chao EY, Cooney WP, Linscheid RL. Forces in the normal and abnormal hand. J Orthop Res 1985;3(2):202-211.

2. Buchholz B, Frederick L, Armstrong TJ. An investigation of human palmar skin friction and the effects of materials, pinch force and moisture. Ergonomics 1988;31(3):317-325.

3. Wells R, Greig M. Characterizing human prehensile strength by force and moment wrench. Ergonomics 2001;4(15):1392-1402.

4. Novo Jr JM. Testes de preensão isométrica da mão: metodologia e implicações fisiológicas. Campinas. [Tese de Doutorado em Engenharia Biomédica - Faculdade de Engenharia Elétrica e de Computação]. Florianópolis (SC): Universidade Estadual de São Paulo;1998.

5. Junichiro Y, Hargens A. Effects of dynamic and static handgrip exercises on hand and wrist volume. Eur J Appl Physiol. 2008;103(1):41-45.

6. Silva ACK. Estudo biomecânico da preensão manual em atletas de diferentes modalidades esportivas. [Dissertação - Centro de Ciências da Saúde e do Esporte]. Florianópolis (SC): Universidade do Estado de Santa Catarina; 2006.

7. Visnapuu M, Jürimäe T. Handgrip strength and hand dimensions in young handball and basketball players. J Strength Cond Res 2007;21(3):923-929.

8. Tsuji S, Tsunoda N, Yata H, Katsukawa F, Onishi S, Yamazaki H. Relation between grip strength and radial bone mineral density in young athletes. Arch Phys Med Rehabil 1995;76(3):234-238.

9. Fry AC, D Ciroslan, Fry MD, Leroux CD, Schilling BK, Chiu LZ. Anthropometric and Performance Variables Discriminating Elite American Junior Men Weightlifters. J Strength Cond Res 2006;20(4):861-866.

10. Budoff, JE. The Prevalence of Rotator Cuff Weakness in Patients with Injured Hands. J Hand Surg 2004:29(6):1154-1159.

11. Álvares-da-silva MR, Silveira TR. Comparison between handgrip strength, subjective global assessment, and prognostic nutritional index in assessing malnutrition and predicting clinical outcome in cirrhotic outpatients. Nutrition 2004;21(2):113-117.

12. Solomon NP, Robin DA. Perceptions of effort during handgrip and tongue elevation in Parkinson's disease. Park Related Disor 2005;11(6):353-361.

13. Voorbij AJM, Steenbekkers LPA. The composition of a graph on the decline of total body strength with age based on pushing, pulling, twisting and gripping force. App Ergonomics 2001;32(3):287-292.

14. Léger L, Boucher R. An indirect continuous running multistage field test: The université de montréal track test. Can J App Sp Sci 1980;5(2):77-84;

15. Jackson A, Dishman RK, Croix S, Patton R, Weinberg $R$. The heart rate, perceived exertion, and pace of the 1.5 mile run. Med Scie Spo Exer 1981;13(4):224-228;

16. Alonso DO, Forjaz CLM, Rezende LO, Braga AMFW, Barreto ACP, Negrão CE, Rondon MUPB. Compor- 
tamento da freqüência cardíaca e da sua variabilidade durante as diferentes fases do exercício físico progressivo máximo. Arq Bras Card 1998;71(6):787-792;

17. Nicolay CW, Walker AL. Grip strength and endurance: Influences of anthropometric variation, hand dominance, and gender. Int J Indus Ergo 2005;35(7):605-618

18. Fess EE. Grip strength. In: Casanova JS, editor. Clinical assessment recommendations. American Society of Hand Therapists. Chicago: 1992, p.41-45.

19. Haidar SG, Kumar D, Bassi RS, Deshmukh SC. Average versus maximum grip strengh: Which is more consistent? J Hand Surg 2004;29(1):82-84.

20. Ruiz-ruiz J, Mesa JLM, Gutiérrez A, Castillo MJ. Hand size influences optimal grip span in women but not in men. J Hand Surg 2002;27(5):897-901.

21. Sande LP, Coury HJCG, Oishi J, Kumar S. Effect of muscoloskeletal disorders on prehension strength. App Ergono 2001;32(6):609-616.

22. Boadella JM, Kuijer PP, Sluiter JK, Frings-Dresen MH. Effect of self-selected handgrip position on maximal handgrip strength. Arch Phy Med Rehab 2005;86(2):328-331.

23. Watanabe T, Owashi K, Kanauchi Y, Mura N, Takahara $\mathrm{M}$, Ogino T. The short-term reliability of grip strength measurement and the effects of posture and grip span. J Hand Surg 2004;30(3):603-609.

24. Bohannon RW, Peolsson A, Massy-Westropp N, Desrosiers J, Bear-Lehman J. Reference values for adult grip strength measured with a Jamar dynamometer: a descriptive meta-analysis. Physiotherapy 2006;92(1):11-15.

25. Claessens A, Buenen G, Lefevre J, Martens G, Wellens R. Body structure, somatotype, and motor fitness of top-class Belgian judoists and karateka. A comparative study. Proceedings of the VIII Commonwealth and In- ternational Conference on Sport, Physical Education, Dance, Recreation, and Health: Kinanthropometry III. Glasgow: London;1986, p.53-57.

26. Little NG. Physical performance attributes of junior and senior women, juvenile, junior, and senior men judokas. J Sports Med Phys Fitness 1991;31(4):510-520.

27. Thomas SG, Cox MH, LeGal YM, Verde TJ, Smith HK. Physiological profiles of the Canadian national judo team. Can J Sport Sci 1989;14(3):142-147.

28. Aziz AR, Tan D, The KC. Physiological responses during matches and profile of elite pencak silat exponents. J Spo Sci Med 2002;1(1):147-155.

29. Warrington G, Ryan C, Murray F, Duffy P, Kirwan JP. Physiological and metabolic characteristics of elite tug of war athletes. J Sports Med 2001;35(6);396-401.

30. Franchini E, Taniko MY, Pereira JNC. Freqüência cardíaca e força de preensão manual durante a luta de jiu-jitsu. Lect Educa Fís Dep. 2003; 65. Disponível em: http://www. efdeportes.com/efd65/jiujitsu.htm. [2005 dez 20].

\section{Endereço para correspondência}

Susana Cristina Domenech

Universidade do Estado de Santa Catarina

Centro de Ciências da Saúde e do Esporte

Laboratório de Instrumentação

Rua Pascoal Simone, 358

Bairro Coqueiros

88080-350 - Florianópolis, SC. Brasil

E-mail: scdomenech@gmail.com 